\title{
Comparative Study between Medial and Posterior Approaches in Management of Popliteal Artery Aneurysm Hany Abdelmomen
}

\author{
Department of Vascular Surgery, Faculty of Medicine, Al-Azhar University \\ Corresponding author: Hany Abdelmomen, Mobile:(+20)01002767339, \\ E-mail: hadydayman@gmail.com
}

\begin{abstract}
Background: Popliteal artery aneurysm (PAA) represents $1 \%$ in men. Bilateral conditions occur in more than $50 \%$ the cases. The most common cause of such aneurysms is atherosclerosis. About $80 \%$ of the PAAs are asymptomatic at the time of diagnosis while $20 \%$ carry high risk for thrombosis and amputation subsequently.

Objectives: This study was conducted to compare the medial and posterior approaches in management of popliteal artery aneurysm repair.

Patients and methods: 40 cases of PAAs were included. Medial approach was done in 20 cases and posterior approach was done to the other 20 cases. Carful history taking and clinical examination was done for proper assessment.

Results: primary patency after 30 day was $100 \%$ with average hospital stay 3.5 days. In the post-operative period, there was few complications with medial approach where one wound hematoma occurred. One asymptomatic graft thrombosis after 11 months occurred. One patient required angioplasty at 24 months with stenting of the distal anastomosis. Graft complications with posterior approach was minimal including one wound seroma, one distal showers in the anterior and posterior tibial arteries at 10 months, one case required balloon angioplasty to the proximal anastomosis at 17 months.

Conclusion: After 6 months, there was no significant difference between the two approaches but if we take in consideration the risk of persistent PAA growth (up to 25\%) after the medial approach, the posterior approach might be the preferred.
\end{abstract}

Keywords: Popliteal artery aneurysm; surgical approach.

\section{INTRODUCTION}

Popliteal artery aneurysm (PAA) is an uncommon problem affecting the vascularity of the lower limb. It represents $1 \%$ in men ${ }^{(1)}$. The age varied from 60 to 85 years. Bilateral conditions occur in more than $50 \%$ of cases. The most common cause of such aneurysms is atherosclerosis, another rare causes include, trauma, infections or familial, in addition to some connective tissue diseases such as Behcet's disease or Marfan's syndrome ${ }^{(2)}$. Two types of popliteal artery aneurysm are present, the first one is confined to the popliteal artery and the second type extends to the superficial femoral artery above the adductor hiatus. About $80 \%$ of the PAAs are asymptomatic at the time of diagnosis. There are many approaches in the management of PAAs ${ }^{(3)}$. Our research included the most common two types of them, medial are posterior approaches.

\section{AIM OF THE WORK}

To compare the medial and posterior approaches in management of popliteal artery aneurysm repair as regards the feasibility of the technique, patency rate, results and complications.

\section{PATIENTS AND METHODS}

Our study was conducted on 40 cases of PAAs in Sayed Galal and AL-Hussein University Hospitals and private hospital through the period between
January 2015 and January 2020. In 20 cases, the medial approach was done, and posterior approach was done to the other 20 cases. Selection of the cases was done on random bases. Carful history taking and clinical

examination was done for proper assessment. In some cases, Doppler and CT angiography was done to confirm that the aneurysm did not extend to the adductor hiatus. Also, to confirm the presence of good distal run-off, otherwise the medial approach was useful for possibility of easy anastomosis proximal to the aneurysm and good thrombectomy of both anterior and posterior tibial arteries. Anesthesia was either spinal or general with endotracheal tube.

\section{Ethical approval:}

The study was approved by the Ethics Board of Al-Azhar University and an informed written consent was taken from each participant in the study. This work has been carried out in accordance with The Code of Ethics of the World Medical Association (Declaration of Helsinki) for studies involving humans.

\section{Operative details}

Written concent was taken from the patient then:

In posterior approach (Figure 1), the patient was positioned in prone position with the knee joint slightly flexed, the whole limb was sterilized by povidone

This article is an open access article distributed under the terms and conditions of the Creative Commons Attribution (CC BY-SA) license (http://creativecommons.org/licenses/by/4.0/) 
iodine, vertical or s-shaped skin incision was done over the popliteal aneurysm across the popliteal fossa provided no healing problems or contracture was done. With the use of such vertical incision, the sural nerve and short saphenous vein was protected. The popliteal vein and medial and lateral popliteal nerves were dissected and proximal dissection and control of the popliteal artery was done and if there is a need. Dissection of the adductor hiatus was done to give more proximal exposure.

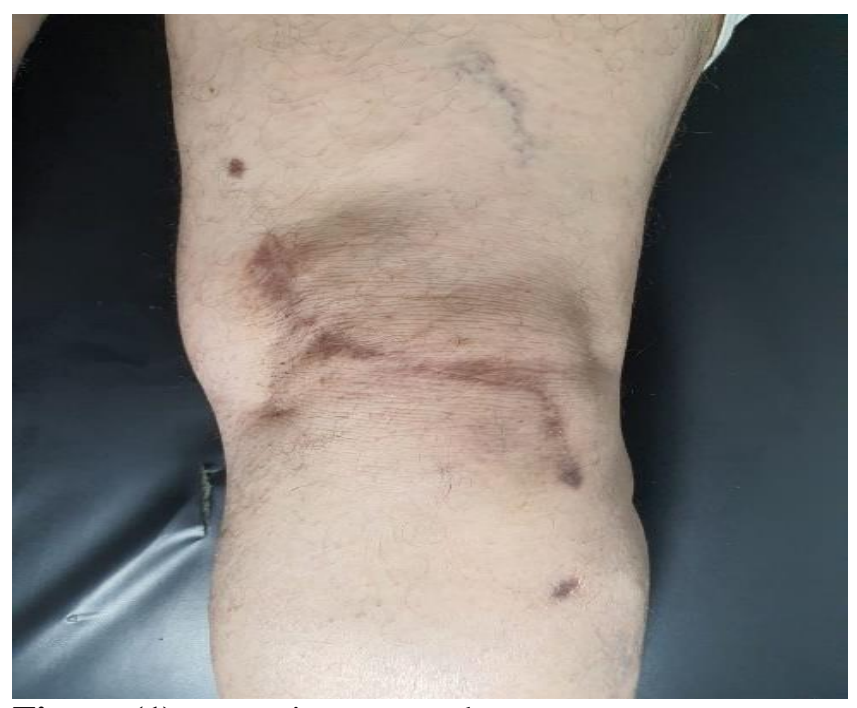

Figure (1): posterior approach

Dissection of the distal popliteal artery and distal control and clamping of the artery was done. $5000 \mathrm{IU}$ of heparin calcium and antibiotics were given, opening of the aneurysmal sac with ligation and suturing of back bleeding points from the openings of genicular arteries. Dacron or PTFE grafts were used with 6 to $8 \mathrm{~mm}$ diameter and proximal and distal anastomosis with 5-0 proline was done with continuous running sutures. We used prosthetic graft but reversed saphenous vein grafts also were performed. The graft was positioned in full extend knee and care was done not to be under tension or taller than the intervening distance.

In medial approach (Figure 2), supine position and sterilization of the limb was done by povidone iodine. Two skin incisions were done, one over the medial supra-genicular aspect and the other on the infra-genicular one. Dissection of popliteal artery and lower superficial femoral artery (SFA) was done through the upper incision. And the lower part of the popliteal and both anterior tibial and posterior tibial arteries through the lower incision. Thrombectomy was done. 5000 IU calcium heparin was given, graft tunneling and insertion and proximal and distal anastomosis by 5-0 proline by continuous suture. Ligation of the native artery above the distal anastomosis, good hemostasis and drain was inserted, then closure of the wounds in layers. The patient was amputated in the next morning with hospital discharge after 3 days.

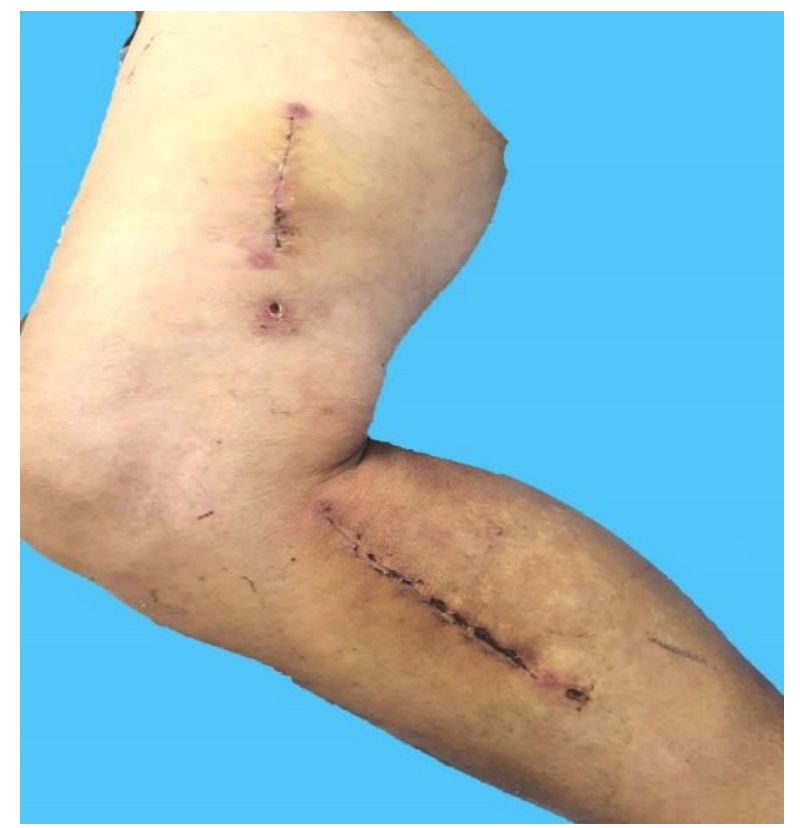

Figure (2): medial approach

\section{RESULTS}

The primary patency rate after 30 day was $100 \%$ with average hospital stay 3.5 days. In the post-operative period few complications occurred with medial approach, one wound hematoma occurred at the infragenicular wound, which was successfully drained after wound opening with carful wash and re-drainage of the wound (Table 1). One graft thrombosis after 11 months in which no intervention was done as the patient was asymptomatic. One patient required angioplasty at 24 months with stenting of the distal anastomosis balloon dilation was successfully done with improvement of the patient's complaint.

Another patient developed aneurysm at the proximal anastomosis at 55 months and managed by removal of the aneurysm and re-anastomosis with the native artery by intervening small synesthetic PTFE. Graft complications with posterior approach was minimal including one wound seroma, which was drained percutaneously. One distal showers in the anterior and posterior tibial arteries at 10 months, which was managed by infra-genicular Thrombectomy and one case required balloon angioplasty to the proximal anastomosis at 17 months.

Another leg paresthesia was present in one case due to sural nerve involvement during surgical management of large aneurysm, which was improved spontaneously after 6 months (Table 1).

Reversed saphenous vein grafts were used in 7 cases, smell vein grafts in 4 cases, synthetic grafts PTFE in 6 cases and Daron grafts in 4 cases in the posterior approach.

In the medial approach, long saphenous reversed grafts in 12 cases, synthetic ringed PTFE grafts in 6 cases and Daron grafts in 2 cases. Long saphenous was used from the ipsilateral limb. 
In the first month postoperative, no deaths were recorded in both groups and no amputation or limb loss was necessary.

Table (1): Complications of both approaches of popliteal artery repair

\begin{tabular}{|l|c|c|}
\hline Complications & $\begin{array}{c}\text { Posterior } \\
\text { group\% } \\
(\mathbf{N = 2 0 )}\end{array}$ & $\begin{array}{c}\text { Medial } \\
\text { group \% } \\
(\mathbf{N = 2 0})\end{array}$ \\
\hline Number of complicatic & 16 & 16 \\
\hline Wound hematoma & 0 & $1(2.5 \%)$ \\
\hline Wound seroma & $1(2.5 \%)$ & 0 \\
\hline Distal ischemia & $1(2.5 \%)$ & 0 \\
\hline Graft thrombosis & 0 & $1(2.5 \%)$ \\
\hline Angioplasty & $1(2.5 \%)$ & $1(2.5 \%)$ \\
\hline Aneurysm & $1(2.5 \%)$ & 0 \\
\hline Leg paresthesia & $0 \%$ & $1 \%$ \\
\hline Amputation & $0 \%$ & $0 \%$ \\
\hline
\end{tabular}

Table (2):

\begin{tabular}{|c|c|c|c|c|}
\hline $\begin{array}{c}\text { Type of } \\
\text { approach }\end{array}$ & $\begin{array}{c}\text { Reversed } \\
\text { long } \\
\text { saghenous } \\
\text { vein graft }\end{array}$ & $\begin{array}{c}\text { Small } \\
\text { vein } \\
\text { graft }\end{array}$ & $\begin{array}{c}\text { PTFE } \\
\text { Graft }\end{array}$ & $\begin{array}{c}\text { Dacron } \\
\text { graft }\end{array}$ \\
\hline $\begin{array}{c}\text { Posterior } \\
\text { approach }\end{array}$ & 7 & 4 & 6 & 4 \\
\hline $\begin{array}{c}\text { Medial } \\
\text { approach }\end{array}$ & 12 & - & 6 & 2 \\
\hline
\end{tabular}

\section{DISCUSSION}

In the bypass with distal and proximal aneurysmal ligation is the most common maneuver (4) because it is easy, safe with easy accessibility of the great saphenous vein, which may be used for natural grafting but this surgical technique may be associated with increase in the size of the aneurysm and development of mechanical complications ${ }^{56,7}$. To overcome this problem, extended medial approach may be used by working around the medial head of gastrocnemius muscle and direct work up on the aneurysm ${ }^{8}$.

The main advantage of posterior approach is definitive exclusion of the PAA. and this is reported by many surgeons but this maneuver having many problems including difficulty in the proximal and distal control that may be present, adherence of the popliteal vein and medial and lateral popliteal nerves to the aneurysm as well as difficulty in harvesting the great saphenous vein from the same wound so we use the short saphenous vein or other medial incision was done to harvest the long saphenous vein ${ }^{9,10}$.

In aneurysms that was extended above the Hunter canal, medial approach was done with extension of the wound towards the medial head of gastrocnemius muscle ${ }^{11}$. In our study we found no significant differences between the medial and posterior approaches in long-term outcomes in PAAs repair not passing the Hunter canal ${ }^{12}$. Both groups were nearly similar in complications, primary and secondary patency rate and limb salvage rates ${ }^{13,14}$. Both groups reaching 6 months without stenosis or occlusion ${ }^{15,16}$.

After 6 months, 2 occlusions occurred in the posterior approach this led to a significant difference between the 2 groups. No neurological complications or problems related to the deep venous system were seen either in the operated limb or the other healthy one were seen in the medial and posterior approaches ${ }^{4}$.

Our primary and secondary patency rates were 90\% compared to Beseth et al. ${ }^{(\mathbf{1})}$ which was $93 \%$ in the primary patency rate and $96 \%$ in the secondary patency rate in the posterior approach, this difference may be due to two early technical failures 1 .

In our study, the graft material also affected the patency rates. The patency rates in saphenous graft was $85 \%$ versus $65 \%$ for PTFE graft $(p<.01)$. The graft material affects the primary and secondary patency rates according to many studies ${ }^{17,18,7}$.

This irrespective of the type of the approach was used. The Major disadvantages of the medial approach is the mechanical increase in size which in some cases required rapid re-intervention. The reported incidence of postoperative aneurysm growth after medial approach is $35 \%$ to $25 \%$ and $50 \%$. This makes necessary to follow-up with duplex ultrasound in cases with aneurysms that extend above the Hunter canal, repair with a medial approach should be done, also as in cases associated with superficial femoral

artery occlusion. ${ }^{1,2,3}$

\section{CONCLUSION}

The medial approach has better primary patency rate ( $<6$ months) compared to the posterior approach and this might be due to the limited field of exposure causing early thrombotic complications, which require re-intervention. Also, the use of autogenous venous graft have higher primary and secondary patency rates compared to PTFE graft irrespective to the type of the approach used.

After 6 months, there is no significant difference between the two approaches but if we take in consideration the risk of persistent PAA growth (up to $25 \%$ ) after the medial approach, the posterior approach might be the preferred surgical approach of PAA repair at the end.

\section{REFERENCES}

1. Beseth B, Moore W (2006): The posterior approach for repair of popliteal artery aneurysms. Journal of Vascular Surgery, 43 (5): 940-5.

2. Aulivola B, Hamdan A, Hile C et al. (2004): Popliteal artery aneurysms: a comparison of outcomes in elective versus emergent repair. Journal of Vascular Surgery, 39 (6): 1171-7.

3. Jones W, Hagino R, Chiou A et al. (2003): Graft patency is not the only clinical predictor of success 
after exclusion and bypass of popliteal artery aneurysms. Journal of Vascular Surgery, 37 (2): 3928.

4. Duffy S, Colgan M, Sultan S et al.(1998): Popliteal aneurysms: a 10-year experience. European Journal of Vascular and Endovascular Surgery, 16 (3): 218-22.

5. Dawson I (2002): Management of popliteal aneurysm. Br J Surg., 89: 1382-1385.

6. van Santvoort $\mathbf{H}$, de Vries J-P, van de Mortel $\mathbf{R}$ et al.(2006): Rupture Of A Popliteal Artery Aneurysm 10 Years After Surgical Repair. Vascular, 14 (4): 22730.

7. Wakassa T, Matsunaga P, Silva E et al. (2006): Follow-up of the aneurysmal sac after exclusion and bypass of popliteal artery aneurysms. Clinics, 61 (2): 107-12.

8. Pulli R, Dorigo W, Troisi N et al. (2006): Surgical management of popliteal artery aneurysms: Which factors affect outcomes? Journal of Vascular Surgery, 43 (3): 481-7.

9. Lowell R, Gloviczki P, Hallett J et al. (1994): Popliteal Artery Aneurysms: The Risk of Nonoperative Management. Annals of Vascular Surgery, 8 (1): 14-23.

10. Kirkpatrick U, McWilliams R, Martin J et al. (2003): Late complications after ligation and bypass for popliteal aneurysm. British Journal of Surgery, 91 (2): $174-7$.
11. Ebaugh J, Morasch M, Matsumura J et al. (2003): Fate of excluded popliteal artery aneurysms. Journal of Vascular Surgery, 37 (5): 954-9.

12. Hirsch A, Haskal $Z$, Hertzer $N$ et al. (2006): ACC/AHA 2005 practice guidelines for the management of patients with peripheral arterial disease. Circulation, 113 (11): e463-e654.

13. Rutherford R, Baker J, Ernst C et al. (1997): Recommended standards for reports dealing with lower extremity ischemia: Revised version. Journal of Vascular Surgery, 26 (3): 517-38.

14. Marty B, Wicky S, Ris H-B et al. (2002): Success of thrombolysis as a predictor of outcome in acute thrombosis of popliteal aneurysms. Journal of Vascular Surgery, 35 (3): 487-93.

15. Blanco E, Serrano-Hernando F, Moñux G et al. (2004): Operative Repair of Popliteal Aneurysms: Effect of Factors Related to the Bypass Procedure on Outcome. Annals of Vascular Surgery, 18 (1): 86-92.

16. Varga Z, Locke-Edmunds J, Baird R (1994): A multicenter study of popliteal aneurysms. Journal of Vascular Surgery, 20 (2): 171-7.

17. Carpenter J, Barker C, Roberts B et al. (1994): Popliteal artery aneurysms: Current management and outcome. Journal of Vascular Surgery, 19 (1): 65-73.

18. Mehta M, Champagne B, Darling $R$ et al. (2004): Outcome of popliteal artery aneurysms after exclusion and bypass: Significance of residual patent branches mimicking type II endoleaks. Journal of Vascular Surgery, 40 (5): 886-90. 